\title{
Multiple Compton scattering gamma ray imaging camera
}

\author{
N. Dogan, D.K. Wehe and G.F. Knoll \\ Department of Nuclear Engineering, University of Michigan, Ann Arbor, MI 48109, USA
}

With the recent advances in position sensing detectors, a compact, multiple layered, Compton scattering gamma ray imaging camera has been investigated for technological feasiblity. Begınnıng from Kamea and Hanada's recent work, the multıple Compton scattering camera design has been investigated and refined over the energy range 150-1000 keV. Our Monte Carlo simulations yielded reconstruction efficiencies of $7-14 \%$ for $\mathrm{S}_{1}$ and $16-28 \%$ for Ge detectors. Including anticipated experimental errors, we obtained angular errors of $-2-5^{\circ}$ in the reconstructed incident gamma ray directions over the energy range of interest.

\section{Introduction}

Gamma ray cameras with good energy and position resolution are needed to locate weak and diffuse radioactive sources and characterize the gamma ray spectra. The concept of Compton scatter imaging and its possible applications were first suggested by Everet [1]. An electronically collimated gamma camera (ECC) was one of the first working cameras based on this principle. The ECC was introduced in 1974, and subsequent work, primarily by Singh, led ultımately to a working prototype for medical imaging [2].

As an extension of the electronic collimation concept, Kamea and Hanada [3,4] introduced a new method called the multiple Compton method. As shown in fig.
1, a multiple Compton camera consists of a series of large area thin silicon (or germanium) layers. Each layer is an independent, two dimensional, position sensitive detector. The layers are designed to be sufficiently thin so the probability of multiple interactions in any layer is small. If the stack is made sufficiently large, there is a reasonable probability that an incoming gamma ray, after a series of Compton scatterings, will be photoabsorbed in one of the layers. Alternately, the stack can be surrounded with a high-efficiency annular detector (a high-density scintillator) to intercept those photons that escape the stack without photoabsorption. Thus, each indıvidual interaction position and energy deposition from either a Compton scattering or photoelectric absorption in the stack is recorded. The energy and

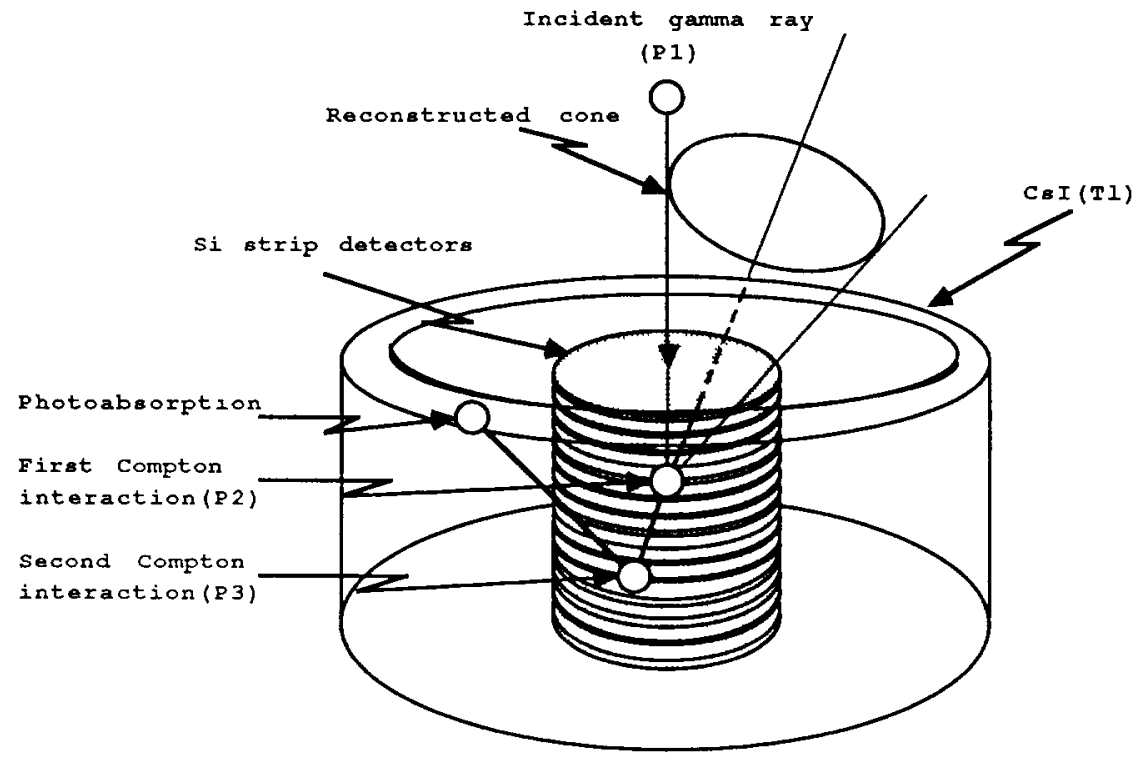

Fig. 1. Schematıc drawing of the multıple Compton camera. 


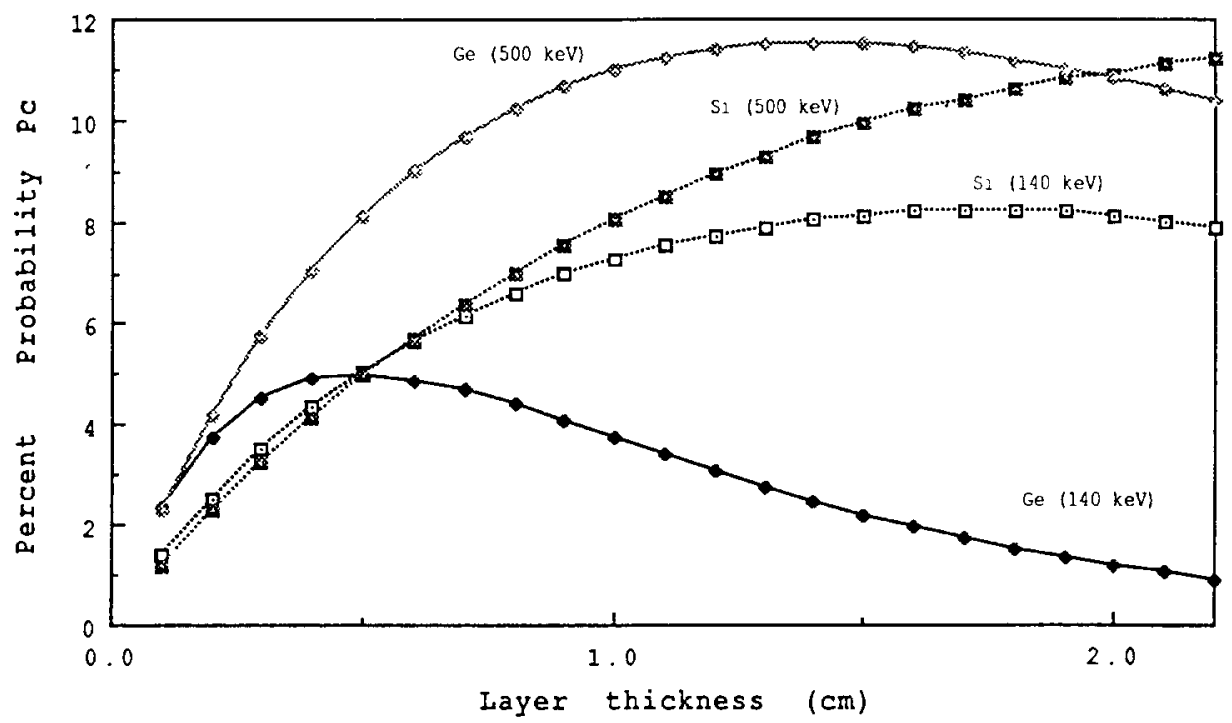

Fig. 2 The probability $P_{c}$ for a single Compton scatterıng event withın a layer and subsequent escape of the scattered gamma ray without undergoing any further interaction in the layer.

azimuthal direction of the incoming photon can usually be obtained from the interaction positions and energy depositions along its history, whether in the stack or in the surrounding annular detector.

While the multiple Compton camera is substantually more complex than the ECC, it offers the potential of high efficiency measurements of the azimuthal direction, polarization, and energy of the incident gamma rays. In this work, we explore the multiple Compton camera concept using Monte Carlo simulations of both $\mathrm{Si}$ and Ge layers.

\section{Evaluation of layer material and thickness}

In order to optımize the type and size of the detectors, computations were performed to determine the corresponding interaction probabilities. The interaction probabilities were calculated for gamma ray photons which are incident perpendicular to the central axis of the stack. The probability of a single Compton scattering event within a layer followed by an escape of the scattered gamma radiation from that layer was calculated from*

$$
\begin{aligned}
P_{\mathrm{c}}(L)= & \int_{0}^{L} \int_{0}^{\pi}\left\{\mathrm{e}^{-\left(\mu_{\mathrm{c}}^{\prime}+\mu_{\mathrm{p}}^{\prime}\right)(L-x) / \cos \theta}\right\}\{P(\theta) \mathrm{d} \theta\} \\
& \times\left\{\mu_{\mathrm{c}} \mathrm{e}^{-\left(\mu_{\mathrm{c}}+\mu_{\mathrm{p}}\right) x} \mathrm{~d} x\right\},
\end{aligned}
$$

* We neglect the effect of the finite radius of the layer and consider escape only through the parallel faces. This approximation will not introduce a significant error in $P_{\mathrm{c}}$ for typical radı being considered. where $L$ is the thickness of a layer, $\mu_{\mathrm{c}}$ and $\mu_{\mathrm{p}}$ are the Compton and photoelectric linear attenuation coefficient of the detector material at the incident gamma ray energy, $\mu_{\mathrm{c}}^{\prime}$ and $\mu_{\mathrm{p}}^{\prime}$ are the corresponding values for the scattered gamma ray energies, $x$ is the depth along the central axis at which an interaction takes place, $\theta$ is the scattering angle for the first Compton interaction, and $P(\theta)$ is the probability of scattering between angles $\theta$ and $\theta+\mathbf{d} \theta$ as obtaned from the Kleın-Nishina formula properly normalized to unity when integration over all angles is carried out.

The values of $\mu_{c}^{\prime}$ and $\mu_{p}^{\prime}$ are computed for each scattering angle using therr energy-dependent behavior [5]. $P_{c}$ was computed by a numerical integration of eq. (1) for $\mathrm{Ge}$ and $\mathrm{Si}$ detectors as a function of layer thickness $L$. As seen in fig. 2, $P_{\mathrm{c}}$ initially increases monotonically with layer thickness for both $\mathrm{S}_{1}$ and Ge, then levels off and decreases above a certain thickness. This behavior is particularly evident at the lower energies for Ge.

In addition to maxımızing $P_{\mathrm{c}}$, we must also minimize the undesirable possibility of multiple scattering with a layer. We have therefore extended eq. (1) to calculate $P_{\mathrm{cc}}$, the probability of observing two consecutive Compton scatterings in a layer for both Ge and $\mathrm{S}_{1}$ layers **. The contributions from more than two scatterings in a given layer were assumed to be small and were ignored.

** This analysis does not include the probability of two scatterıngs in a given layer which are separated by one or more scatterıngs in other layers. Monte Carlo studies have shown that including this introduces a negligible increase in $\mathrm{P}_{\mathrm{cc}}$. 
Fig. 3 shows the ratio of $P_{\mathrm{cc}} / P_{\mathrm{c}}$ as a function of detector thickness for different gamma ray energies for both $\mathrm{Ge}$ and Si. For a layer thickness of $5 \mathrm{~mm}, P_{\mathrm{cc}}$ is at least an order of magnitude smaller than $P_{\mathrm{c}}$ for both $\mathrm{Si}$ and Ge. We therefore conclude that multiple scatterings within a layer will not complicate the image formation provided layers are kept smaller than several millimeters.

\section{Image formation from the interaction data}

In order to illustrate the multiple Compton method, consider a gamma ray emitted from $\mathrm{P}_{1}$ in fig. 1 and incident normally to the camera. Assuming the first interaction occurs at $P_{2}$, the recoil electron will deposit energy $\Delta E$, a part of the incident energy $E_{0}$, in the vicinity of the interaction. The scattered gamma ray will change direction by some angle $\theta_{1}$, and will normally undergo further interactions in the semiconductor stack. In the energy range of interest and using silicon as the detector material, a typical gamma ray will scatter 3-5 times in the camera before finally undergoing photoabsorption. In order to reconstruct the direction of the incident photon, only the details of the first two interactions are needed. If the gamma ray is totally absorbed in the camera, one can show the incident direction is given by:

$\theta=\cos ^{-1}\left(\frac{E_{0}^{2}-E_{0} \Delta E-m_{0} c^{2} \Delta E}{E_{0}^{2}-E_{0} \Delta E}\right)$,

where $\theta$ is measured relative to the line defined by two interaction points, and $\Delta E$ is the energy deposition in the first interaction. If the gamma ray is not totally absorbed within the camera, the first three interactions are sufficient to resolve the incident energy and direc- tion. In practice, the difficulty is to identify the correct sequence of the interactions in the stack. Since interactions are separated in time by less than a nanosecond, direct timing information is not available to unambiguously identify the specific sequence. However, the known kinematics of Compton scattering can be used to resolve this ambiguity for most cases.

The sequence reconstruction process can be illustrated by considering the case of a gamma ray which undergoes $N-1$ scatterings before being photoabsorbed in the stack. The incident energy $E_{\text {total }}$ is calculated from the summed energy depositions $\Delta E_{J}$ at all $N$ interaction points. One then randomly chooses one of the $N$ interaction points and assumes that it is the first. There are $(N-1)$ remaining candidates to select as the next interaction point. Each of these defines a specific direction and energy deposition. For an assumed sequence, the energy and momentum of the initial and final gamma rays and deposited energy can be calculated for each Compton scattering. For the $j$ th interaction, energy and momentum conservation yields:

$$
\begin{aligned}
& E_{J}^{\text {initial }}=E_{\text {total }}-\sum_{i=1}^{\prime-1} \Delta E_{\iota}, \\
& E_{J}^{\text {final }}=E_{J}^{\text {intial }}-\Delta E_{J}, \\
& \theta_{J}=\cos ^{-1}\left\{1+m_{0} c^{2}\left(\frac{1}{E_{J}^{\text {intial }}}-\frac{1}{E_{J}^{\text {final }}}\right)\right\} .
\end{aligned}
$$

The calculated $\theta$ is compared with the actual angle between the two line segments $\mathrm{P}_{j-1} \mathrm{P}_{j}$ and $\mathrm{P}_{j} \mathrm{P}_{\jmath+1}$ (within an acceptance limit $\delta$ ) to determine the validity of the assumed sequence. Most tests could be terminated quickly because the necessary scattering angle is not consistent with the measured energy deposition. Once all possibilities have been eliminated for a specific ini-

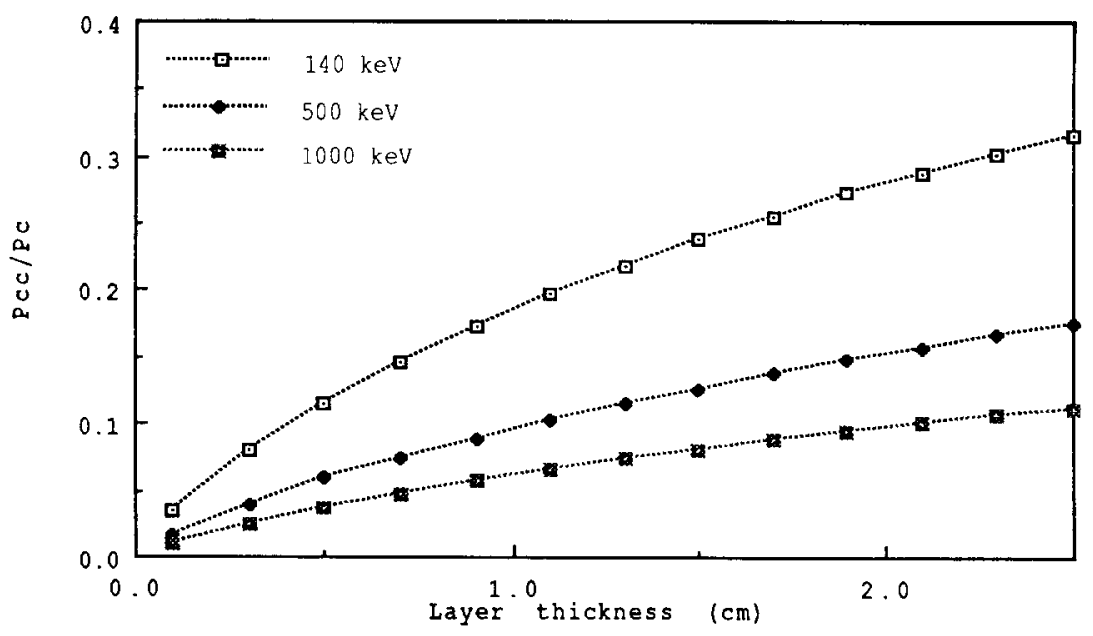

Fig. 3 Ratio of double Compton scattering to single Compton scattering in a Si layer. 


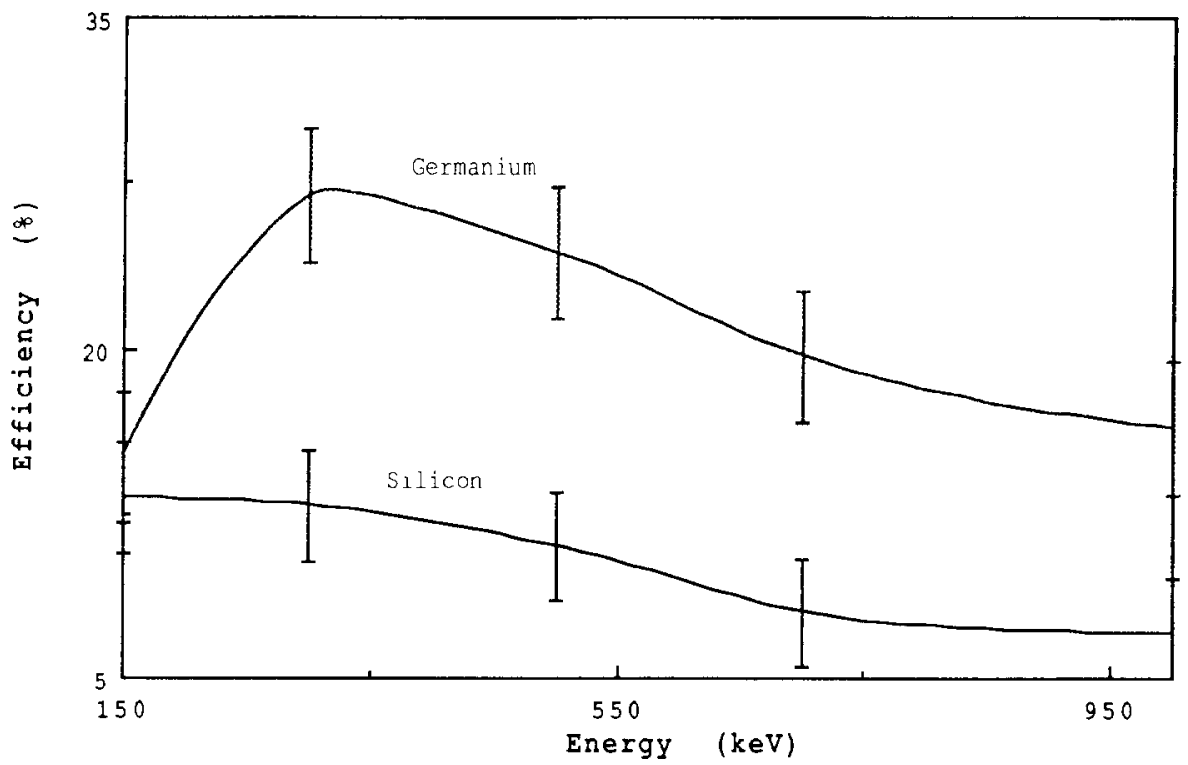

Fig 4 Reconstruction efficiency versus energy of the incident gamma ray for Si and Ge.

tial choice, that choice is discarded as a candidate and another is made. The tests are repeated until only the correct sequence survives. The sequence reconstruction procedure yields a unique incident azimuthal angle, but offers no information on the incident polar angle which can vary from 0 to $2 \pi$.

Therefore, the possible directions of the incident photon define a cone. The point where the first Compton scattering occurred serves as the apex of the cone, while the direction of the scattered gamma ray lies on the symmetry axis of the cone. The conical shape results because any incident gamma ray travelling on the cone surface may scatter through the same azimuthal angle $\theta$ and yield identical energy depositions. For each sequence, we calculate the cone on which the incident gamma ray lies and the thickness of the cone caused by the assumed measurement error. Within the thickness of the cone, a probability density is defined. The source intensity distribution is then reconstructed by superposition of these probability densities from multiple incident gamma ray photons. A single point source will be reconstructed as a broadened point superimposed on a featureless background due to the polar ambiguity of each direction determination.

\section{Computer simulation results}

The sample camera design shown in fig. 1 has been tested using the Integrated Tiger Series (ITS) package of Monte Carlo codes [6]. The three basic codes which form the ITS package differ primarly in their dimensionality and sophistication in geometric modelling. We have used the two-dimensional CYLTRAN code which sımulates Compton scattering, Rayleıgh scattering and the photoelectric effect over the range from $1 \mathrm{keV}$ to several MeV. Each simulation used a batch of 10000 gamma rays incident normally to the camera and distributed over the energy range $150-1000 \mathrm{keV}$. The output from each Monte Carlo run yielded the interaction positions and energy depositions associated with each incident gamma ray history. In addition, the sequence of these interactions was also recorded for comparison with the results of the sequence reconstruction algorithm. We also introduced an uncertainty in position measurement equal to one half the layer thickness, consistent with experience using silicon strip detectors $[7,8]$. No uncertainty was assumed for the energy measurements. The output from the Monte Carlo simulations was used as input to the reconstruction methodology described in section 4 .

Fig. 4 shows the reconstruction efficiency * of the sample design for both Ge and Si detectors for a point source located on the central axis of the semiconductor detector $5 \mathrm{~cm}$ from the face and over the energy range $150-1000 \mathrm{keV}$. The proposed design yields reconstruction efficiencies 7-14\% for $\mathrm{Si}$ and $16-28 \%$ for $\mathrm{Ge}$ detectors. This high efficiency implies the camera will have sufficient sensitıvity to produce umages of relatively weak radioactive sources. Note that at higher energies, choosing germanium detectors improves the

\footnotetext{
* The reconstruction efficiency is defined as the fraction of incident photons whose incident direction and energy are correctly reconstructed. For example, the incident energy and direction of a photon having only one interaction in the stack cannot be reconstructed.
} 


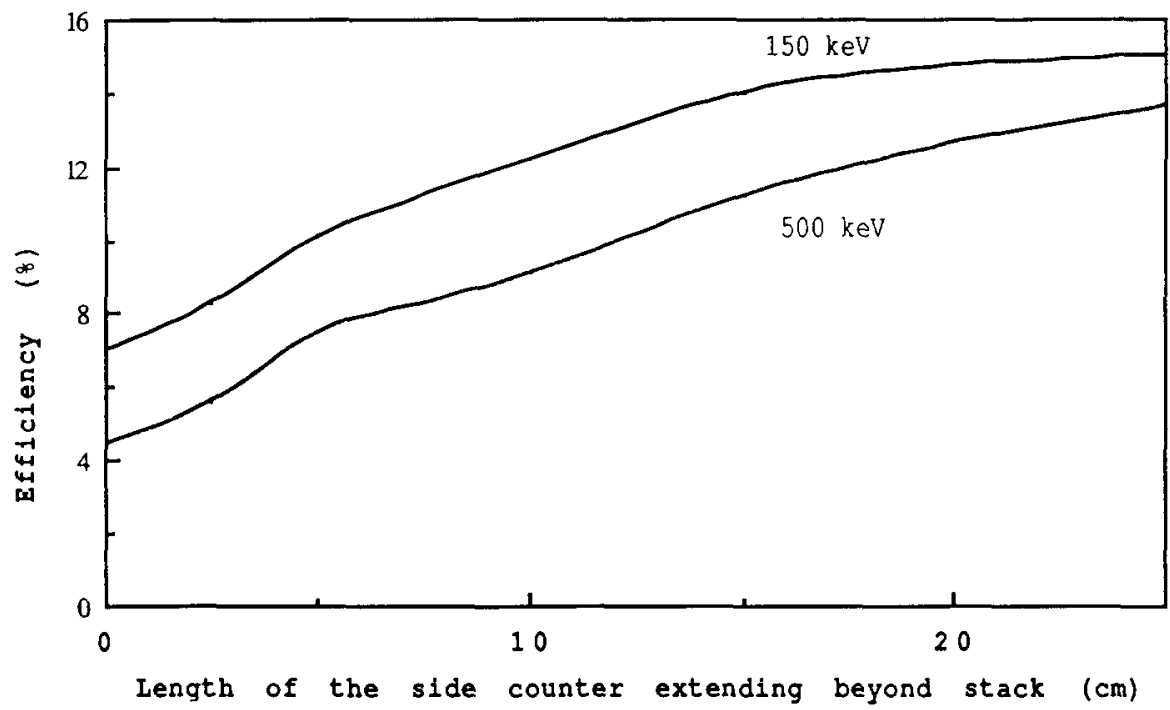

Fig. 5. Variation of reconstruction efficiency with extension of the side counter beyond the stack for 150 and $500 \mathrm{keV}$ gamma rays in Si.

performance dramatically, while below $200 \mathrm{keV}$ silicon offers comparable efficiency. Figs. 5 and 6 show the changes in the reconstruction efficiencies with changes in the length of the annular side counter and layer areas for gamma ray energies of $140-500 \mathrm{keV}$. Fig. 5 indicates the substantial gain in reconstruction efficiency by extending the side counter well beyond the length of the stack. Since the side counter is relatively inexpensive relative to the stack components, increasing its size appears to be an efficient means of increasing the camera sensitivity. Fig. 6 shows similar results for increasing the radii of the layers.

\section{Conclusions}

The design and feasibility of a multiple Compton camera using $\mathrm{Si}$ or Ge microstrip detectors and CsI as a surrounding annular detector has been investigated in the energy range $150-1000 \mathrm{keV}$. The results provide the

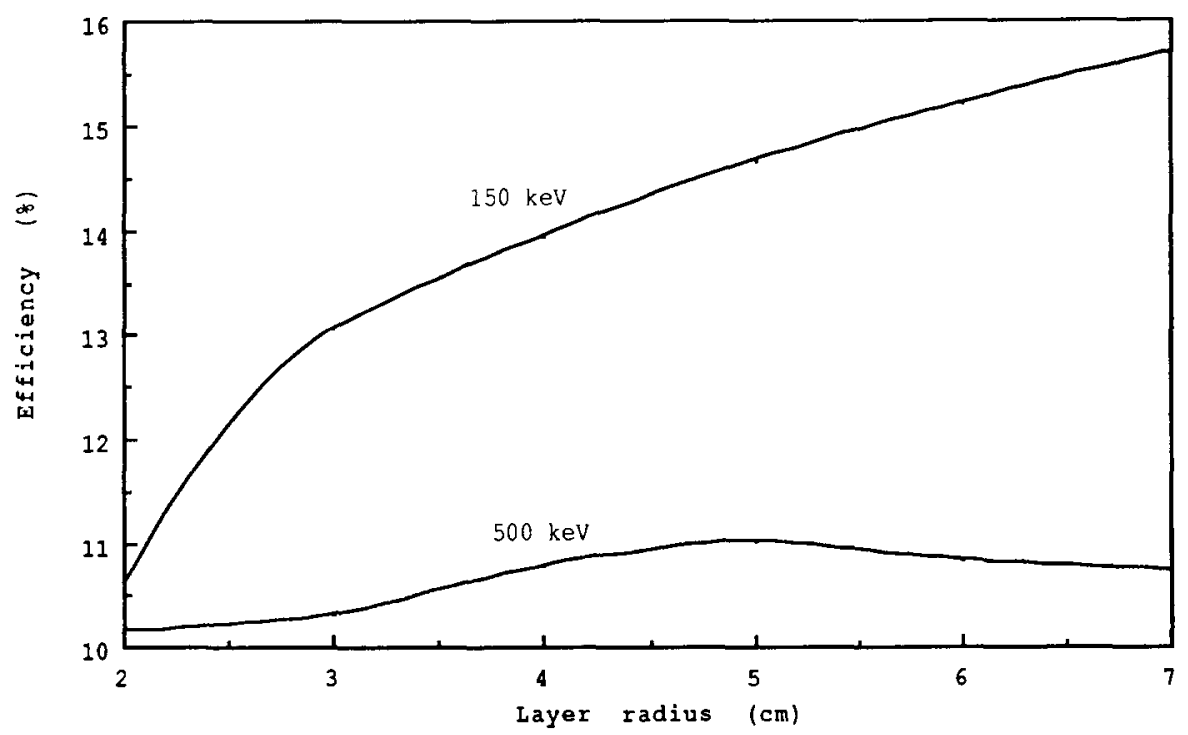

Fig. 6. Variation of the reconstruction efficiency with layer radıus for 150 and $500 \mathrm{keV}$ gamma rays in Si. 
guidance for choosing the camera's design parameters. Choosing layer thicknesses of $-5 \mathrm{~mm}, 2.5 \mathrm{~cm}$ radii, and an extended side counter results in a camera with a high reconstruction efficiency for incident gamma rays $\leq 1 \mathrm{MeV}$. Furthermore, these parameters are feasible with the emerging developments in silicon strip detectors and semiconductor drift detectors. We believe this new camera concept will find a wide variety of applications in imaging gamma ray distributions. The relatively small size of the camera will be an advantage partıcularly in industrial settings where portability is required and low intensities are common.

\section{Acknowledgements}

The authors would like to express their appreciation to the INEL Supercomputing Center (Dick Curtis),
Cray Research Inc. (Steve Baumann), and the Department of Energy for providing the resources necessary to perform this work.

\section{References}

[1] D.B. Everet et al., Proc. IEEE vol. 124 (1977) p. 995.

[2] M. Singh and D. Doria, Med. Phys. 10 (1983) 421.

[3] T. Kamea et al., Nucl. Instr and Meth. A260 (1987) 254.

[4] T Kamea and H. Hanada. IEEE Trans. Nucl. Scı. NS-35 (1) (1988) 352.

[5] I. Kaplan, Nuclear Physics 2nd ed. (Addison-Wesley, 1963) pp. 403-406

[6] A. Halblesb and T.A Melhorn, The Integrated TIGER Sernes of Coupled Electron/Photon Monte Carlo Transport Codes, Sandia National Laboratory (1984).

[7] J.T. Walton, Nucl. Instr. and Meth 226 (1984) 1.

[8] E. Belau et al., Nucl. Instr. and Meth. 214 (1983) 253. 